\title{
Editorial
}

\section{Information and Modeling in Complexity 2013}

\author{
Carlo Cattani, ${ }^{1}$ Shengyong Chen, ${ }^{2}$ and Gani Aldashev ${ }^{3}$ \\ ${ }^{1}$ Department of Mathematics, University of Salerno, Via Ponte Don Melillo, 84084 Fisciano, Italy \\ ${ }^{2}$ School of Computer Science, Zhejiang University of Technology, Hangzhou 310023, China \\ ${ }^{3}$ Department of Economics, University of Namur, Rempart de la Vierge 8, 5000 Namur, Belgium
}

Correspondence should be addressed to Carlo Cattani; ccattani@unisa.it

Received 5 August 2013; Accepted 5 August 2013

Copyright (c) 2013 Carlo Cattani et al. This is an open access article distributed under the Creative Commons Attribution License, which permits unrestricted use, distribution, and reproduction in any medium, provided the original work is properly cited.

Recent research in engineering systems builds theories represented by mathematical models that aim at understanding fundamental questions of spatial structure, self-organization, environmental interaction, behavior, and development. Thus, researchers and engineers are increasingly facing the challenge of dealing with complexity in practical applications that are based on efficient mathematical models.

The topics that deal with these issues can be divided into two parts: (i) development of general mathematical methods/models and (ii) specific applications in particular domains. The focus of this issue on topics of information and modeling complexity is both on the mathematical models and the engineering applications in complex systems. Moreover, we are interested in both new theoretical developments and the studies of practical implementation concerning modeling, complexity, fractals, statistics, and signal extraction and transformation.

Complex systems, such as living systems, are often maintained by information flows. Signals extract such information from the complex phenomena being measured. Such signals are typically a time series having both a regular and a random component. Solutions of the arising mathematical problems attempt to map general principles for modeling how the complex systems operate. This area is a burgeoning field of research due to both the practical significance of the applications and the general scientific importance.

Further to the 63 submissions in 2012, the special issue receives 94 new submissions in 2013. After a rigorous review process, 36 papers, that is, a $38.3 \%$ acceptance rate, which represent a good panel in the related new development, are selected in the journal, with the topics being connected with modeling of complex systems, computational issues in dynamical systems, signal processing of complex information, complexity and parameters, entropy, multifractality, Hurst exponent, correlation, power spectrum, and practical applications.

In the category of modeling, M. Scalia et al. study the Johnson noise in biological matter, $\mathrm{L}$. Wu et al. propose a contextual hierarchical part-driven conditional random field model for object category detection, and M. Li and W. Zhao investigate on 1 over $f$ noise. L. Zhu et al. propose a bilayer resource model for cloud manufacturing services. L. Wu et al. study the inverse 1-median problem on tree networks with variable real edge lengths. L. Luo et al. present maximum variance hashing via column generation. J. Hu et al. show the adaptive multilevel kernel machine for scenic classification. X. Duan et al. propose a Shannon-Runge-Kutta-Gill method for convection-diffusion equations. $\mathrm{H}$. Ma et al. present strong list edge coloring of subcubic graphs. J. Leng et al. construct bivariate nonseparable compactly supported orthogonal wavelets. Z.-W. Li et al. propose a dynamically weighted clique evolution model in clique networks. B. Nouhi et al. study chaotic charged system search with a feasible-based method for constraint optimization problems. L. Huang et al. propose a dynamics-based stranded-crowd model for evacuation in building bottlenecks. E. G. Bakhoum and C. Toma make modeling transitions in complex systems by multiplicative effect of temporal patterns extracted from signal flows.

On computational issues, M. Aktas and E. Agcakoca study the impact of the HMCFRP ratio on the strengthening of steel composite I-beams. M. Li represents a smoothed spectrum estimate with the Cauchy integral. S. Liu et al. show approximation slack scaling for structural support 
vector machines in scene depth analysis. C. Biscarini et al. make detailed simulation of complex hydraulic problems with macroscopic and mesoscopic mathematical methods. D. Sendrescu proposes parameter identification of anaerobic wastewater treatment bioprocesses using particle swarm optimization. M. Chen et al. construct a cloud computing platform for an online model library system. Z. Lu et al. study normality of Ethernet traffic at large time scales. X. Huang et al. propose a marching cubes algorithm for fast modeling of human face by incremental data fusion. P.-G. Popescu et al. present new inequalities between information measures of network information content. X. Xu et al. give a hybrid discrete differential evolution algorithm for lot splitting with capacity constraints in flexible job scheduling. H. Pan et al. review recent modeling for deformable body and motion analysis. K. Guo et al. propose a differential and statistical approach to partial model matching. J. Zhang et al. propose a multiobjective quantum evolutionary algorithm for the vehicle routing problem with customer satisfaction.

On practical applications, $\mathrm{Z}$. Lu et al. give stationarity testing of accumulated Ethernet traffic. Y. Niu et al. work on airborne infrared and visible image fusion for target perception based on target region segmentation and discrete wavelet transform. F. Su et al. identify adaptive color features in image for object tracking. D. Zhang et al. show human model adaptation for multiview markerless motion capture. Y.-S. Juang et al. make fast texture synthesis in adaptive wavelet packet trees. F.-H. Cheng and T.-Y. Sung merge static and dynamic depth cues with optical-flow recovery for creating stereo videos. A. Marucci et al. study energy efficiency of a greenhouse for the conservation of forestry biodiversity. $\mathrm{H}$. Cai et al. propose entropy-based maximally stable extremal regions for robust feature detection. C. B. Guure and N. A. Ibrahim use Bayesian analysis of the survival function and failure rate of Weibull distribution with censored data.

Nevertheless, this special issue is not intended to constitute a complete compendium on the topic, but it does offer the reader an opportunity to appreciate the possibilities and the progress in the research area. It can be seen that although some models and solutions become available, most problems remain open and research is highly active in this field. We are aware of the present and upcoming research progress in the field.

\section{Acknowledgments}

As guest editors of this special issue, we would like to thank all the authors for their valuable contributions and the referees for their thorough reviews and helpful comments on the papers. 


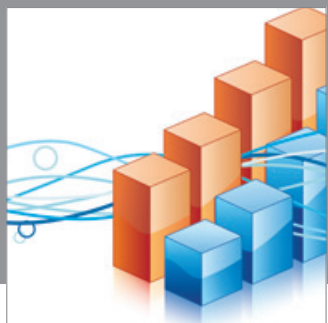

Advances in

Operations Research

mansans

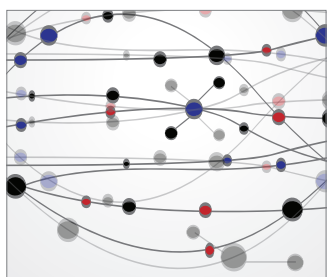

The Scientific World Journal
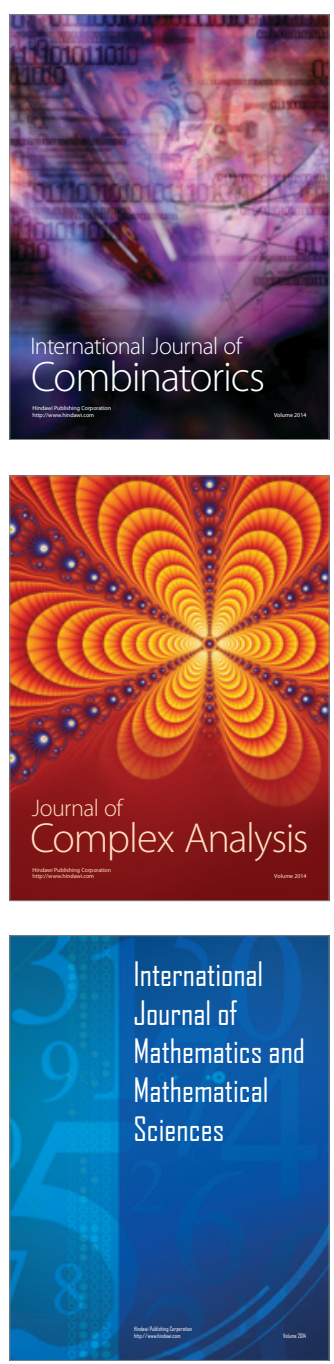
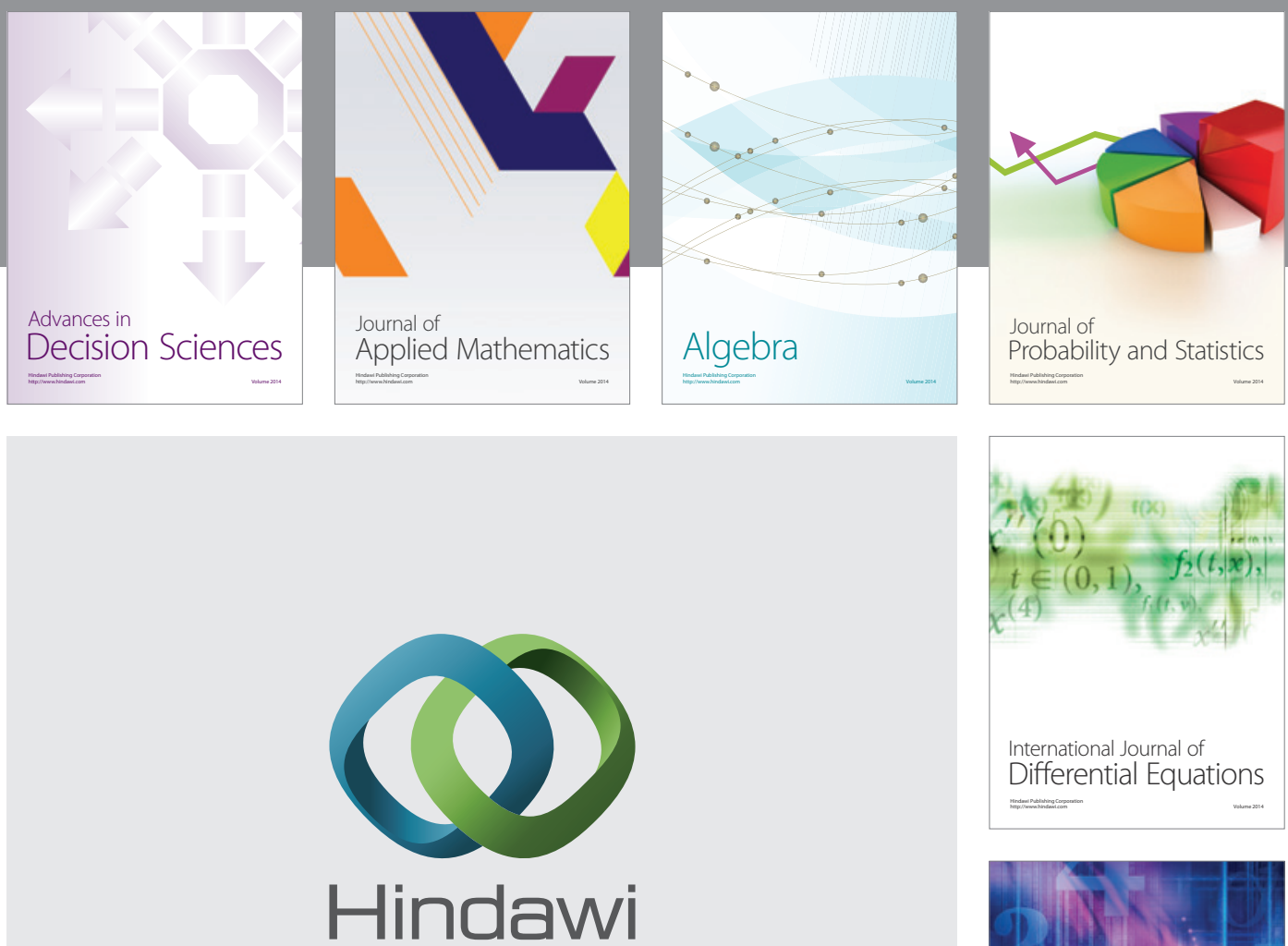

Submit your manuscripts at http://www.hindawi.com
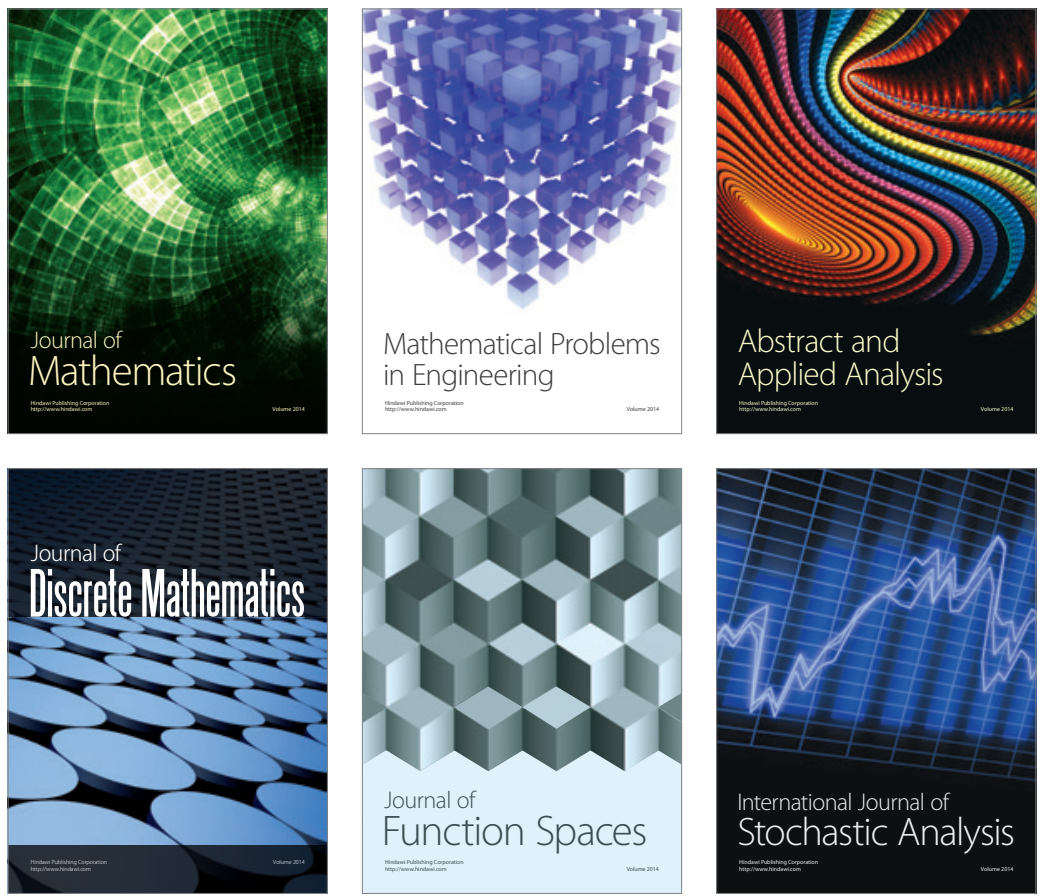

Journal of

Function Spaces

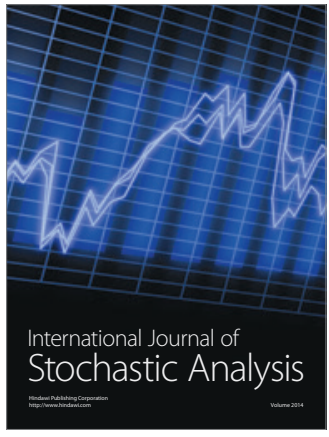

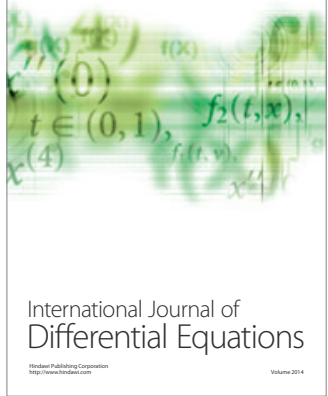
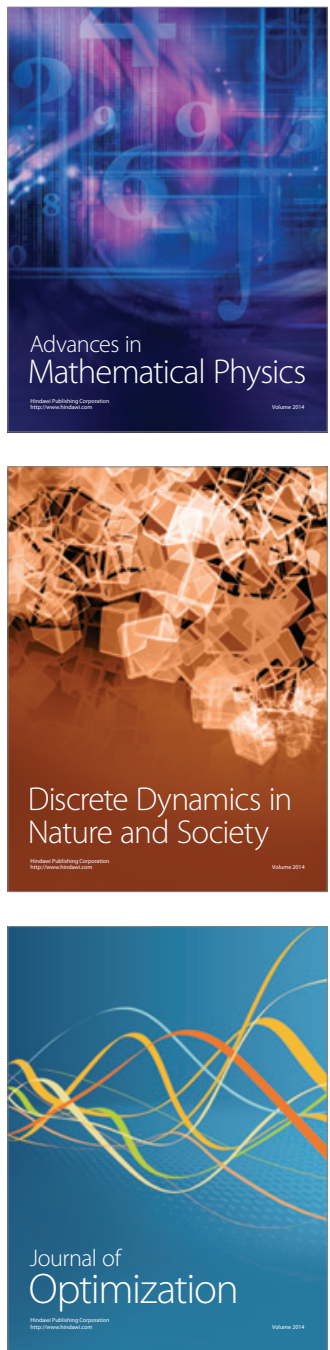